\title{
Comparison of TRUS and Combined MRI Targeted plus Systematic Prostate Biopsy for the Concordance Between Biopsy and Radical Prostatectomy Pathology
} \author{
Group $^{1}$ \\ ${ }^{1}$ Affiliation not available \\ ${ }^{2}$ Izmir Bozyaka Training and Research Hospital \\ ${ }^{3}$ Gazi University \\ ${ }^{4}$ Hacettepe University \\ ${ }^{5}$ Cukurova University Faculty of Medicine \\ ${ }^{6}$ Acibadem University
}

Guven Aslan ${ }^{1}$, Serdar Çelik ${ }^{2}$, Sinan Sözen ${ }^{3}$, Bulent Akdogan ${ }^{4}$, Volkan Izol ${ }^{5}$, Cenk Yucel Bilen $^{4}$, Bahadır Şahin ${ }^{1}$, Levent Turkeri ${ }^{6}$, and Members of Urooncology Association Study

October 1, 2020

\begin{abstract}
Aim: To evaluate the accuracy in histologic grading of MRI/US image fusion biopsy by comparing conventional 12-core TRUS$\mathrm{Bx}$ at radical prostatectomy specimens (RP). Methods: Consecutive patients diagnosed prostate cancer (127 with combination of both targeted biopsy (TBx) plus systematic biopsies (SBx) and separate patient cohort of 330 conventional TRUS-Bx without mpMRI) with a PSA level of $<20 \mathrm{ng} / \mathrm{ml}$ prior to RP were included. The primary end point was the grade group concordance between biopsy and RP pathology according to biopsy technique. Results: Clinically significant prostate cancer detection was $51.2 \%$ for TRUS-Bx, $49.5 \%$ for SBx, $67 \%$ for TBx and $75.7 \%$ for TBx+SBx. Upgrading and downgrading of at least one Gleason Grade Group (GGG) was recorded in 43.3\% / 6.7\% patients of the TRUS-Bx, and in 20.5\%/22 \% of the TBX+SBx group, respectively (all $\mathrm{p}<0.001$ ). Concordance level was detected to be significantly higher for ISUP 1 in combined TBx $+\mathrm{SBx}$ method compared to conventional TRUS-Bx $(61.3 \%$ vs $37.9 \%, \mathrm{p}=0.014)$. In ISUP 1 exclusively, significant upgrading was seen in TRUS-Bx (62.1\%) when compared to TBx (41.4\%) and TBx+SBx (38.7\%). Conclusions: MRI-targeted biopsies detected more significant PCa than TRUS-Bx but, superiority in significant cancer detection appears as a result of inadvertant selective sampling of small higher grade areas. within an otherwise low grade cancer and does not reflect accurate GGG final surgical pathology. TBx $+\mathrm{SBx}$ has the greatest concordance in ISUP Grade 1 with less upgrading which is utmost important for active surveillance.
\end{abstract}

\section{What is known?}

MRI targeted biopsies are reported to be able to better detect high grade cancers than systematic biopsies and TRUS. However, several recent studies have reported a mismatch in the biopsy and radical prostatectomy Gleason Score. Despite the improved detection of clinically significant cancers with MRI targeted biopsies, considerable disparity exists in the literature regarding MRI targeted biopsy's ability to better detect RP pathology in biopsy-naive men over conventional TRUS biopsies.

\section{What is new?}

Combined $\mathrm{TBx}+\mathrm{SBx}$ provides improved detection rates particularly for higher-grade disease over either 
systematic or MRI-targeted biopsy or TRUS-Bx alone. However, superiority in significant cancer detection did not reflect accurate Gleason Grade Group in whole prostate gland at final surgical pathology due to selective sampling of higher grade areas within an otherwise low grade cancer. There is a risk of overtreatment if biopsy GGG was taken as the sole parameter for treatment decision by TBx $+\mathrm{SBx}$. Conventional TRUSBx has superior concordance with radical prostatectomy for ISUP Grade 2,3,5 however increased upgrading should be taken into account.

Key words: Prostate cancer, targeted biopsy, MRI/US Fusion, prostate biopsy, concordance, upgrading, downgrading

\section{Introduction}

Twelve core transrectal ultrasound guided prostate biopsy (TRUS-Bx) is the most commonly used method for the diagnosis of prostate cancer $(\mathrm{PCa})$. However, it may be hampered by several limitations such as not being targeted and not fully anatomical systematic biopsy method when we look at the magnetic resonance imaging (MRI) studies (1-3). This method is also shown to be associated with missed diagnosis of PCa, misclassification of cancer grade and high upgrading and downgrading levels after RP $(4,5)$. Upgrading can in fact lead to inappropriate or under-treatment in a subgroup of patients. A more accurate diagnostic method is crucial to avoid misclassification, which is particularly important in appropriate decision-making for the treatment of $\mathrm{PCa}$ such as active surveillance or other therapies. Prior researches have shown that multiparametric MRI targeted biopsy identifies clinically significant prostate cancers more accurately than conventional systematic biopsy in men with suspected localized prostate cancer (6-9). Despite the improved detection of clinically significant cancers with MRI targeted biopsies, debate persists about whether MRI targeted biopsy better predicts final pathology at radical prostatectomy $(10,11)$. The MRI targeted biopsy results in the previous studies reported that targeted biopsies have a higher rate of concordance level with the RP compared to systematic biopsy $(6-8,12-14)$. However, there is limited data comparing to head to head concordance, upgrading and downgrading between conventional TRUS-Bx and MRI Fusion biopsies according to ISUP grade groups. Whether MRI/US image fusion biopsy techniques better correlate with final histopathologic outcomes by reducing Gleason Score misclassifications deserves further evaluation.

In this context we aimed to analyze the ISUP Gleason group grading concordance between prostatectomy and biopsy stratified by the approach of biopsy technique (TBx $+\mathrm{SBx}$ vs TRUS-Bx). Optimal agreement between biopsy and surgical Gleason Score among three biopsy schemes TBx alone vs SBx alone vs combined $\mathrm{TBx}+\mathrm{SBx}$ is also investigated.

\section{Material and Methods}

We retrospectively reviewed completely anonymized data from nation-wide tertiary centers in Prostate Cancer Database of Turkish Urooncology Association. MRI targeted biopsy is limited yet in this country and several centers are using conventional 12-core transrectal ultrasound-guided prostate biopsy (TRUS-Bx) protocol without mpMRI for the diagnosis of prostate cancer. Therefore our prostate biopsy database has two different data entry: first separately from conventional TRUS-Bx without mpMRI and second MRI fusion biopsy (combined targeted and concomitant systematic biopsies) registered accordingly from collaborating centers in our database. Biopsy naive patients who were diagnosed with prostate cancer either with conventional TRUS-Bx or MRI-Targeted biopsy combined with systematic 12-core biopsy (TBx $+\mathrm{SBx})$ and underwent radical prostatectomy (RP) between 2017-2020 were evaluated in this study. Cognitive MRI fusion biopsies were excluded. Patients who had PSA level less than $20 \mathrm{ng} / \mathrm{ml}$ were included in the study to exclude high grade advanced PCa to ensure proportional distribution of PCa grades between biopsy methods. Patients who had complete pathological data of each Bx scheme and data of RP were investigated. The patients' age, PSA level, the highest GS and ISUP grades from each Bx scheme, RP pathology and upgrade/downgrade ratios were assessed. Patients were divided into two groups as Combined TBx $+\mathrm{SBx}$ and conventional TRUS-Bx method. Data were compared between these groups. Also, a sub-group analysis of the concordance of ISUP grades, upgrade, downgrade ratios between TBx alone, SBx alone and combination $\mathrm{TBx}+\mathrm{SBx}$ scheme was investigated. 
All TRUS-Bx biopsies were taken with a transrectal approach under local anesthesia using the 12 core approach. All mpMRI for targeted biopsies were reviewed by a specified institutional radiologist. In patients with a PI-RADS-lesion [?]3 (according to PI-RADS-v2 classification) (15), MRI Targeted ultrasound fusion biopsy using different software-based platforms according to participant center's property MIMS Symphony Dx@ (MIM Software), bk3000@ (BKMedical), UroNav@ (Invivo Corp, Philips) was conducted. MRI image fusion targeted biopsies were taken from each target with at least 2 or more core samples from each target lesion. In addition to targeted biopsies, systematic random biopsies were also performed using the 12-core approach.

The biopsy GS was defined as the highest Gleason score in at least one core and was reported using the ISUP Consensus Conference 2014 grading system (16).

\section{Statistical analysis}

Study data were gathered and managed using REDCap electronic data capture tools hosted at Urooncology Association Turkey $(17,18)$. REDCap (Research Electronic Data Capture) is a secure, web-based software platform designed to support data capture for research studies. The T-test, Mann-Whitney U test and Chisquare test were used to analyze the relationship of categorical and continuous variables between two biopsy methods and three biopsy schemes of the combined biopsy method. A p value less than 0.05 were considered statistically significant.

\section{Results}

Data from 457 patients who underwent RP after the diagnosis of $\mathrm{PCa}$ in 127 patients with combined TBx + SBx and 330 with conventional TRUS-Bx method were evaluated. Patient demographics of the study cohort stratified by biopsy approach are depicted in Table 1. Mean age and PSA values between the groups were similar. The median number of targeted biopsy cores sampled per region of interest (ROI) was 4 (range 2-7).

In the comparison of biopsy ISUP grades between TRUS-Bx and targeted biopsies, higher ISUP grades were detected by combined TBx+SBx. Significant cancer detection was $49.5 \%$ for SBx, $67 \%$ for TBx and highest $75.7 \%$ for Combination TBx $+\mathrm{SBx}$. There was significant difference in missed cancer ratio between TBx and SBx (10.2\% vs $24.4 \%)$ that any grade cancer detection by SBx alone was significantly lower than TBx alone.

ISUP distributions and upgrade / downgrade ratios of the methods are also given in Figures 1,2 and Tables 1. Overall Biopsy and surgical pathologic ISUP Grade were concordant in $50 \%$ men of the TRUS-Bx and in $57.5 \%$ of the combined TBX $+\mathrm{SBx}$ group $(\mathrm{p}=0.152)$. Gleason upgrading / downgrading of at least one Gleason Grade Group (GGG) was recorded in $43.3 \%$ / $6.7 \%$ patients of the TRUS-Bx, and in $20.5 \% / 22 \%$ of the TBX $+\mathrm{SBx}$ group, respectively (all $\mathrm{p}<0.001$ ).

In the evaluation of conventional TRUS-Bx method according to ISUP grades, concordance achieved in $37.9 \%, 70 \%$, and $56.1 \%$, in ISUP 1, 2 and 3 grades respectively, at RP pathology. In the TRUS-Bx group, after prostatectomy significant upgrading of $62.1 \%$ was detected in ISUP 1 grade.

For ISUP 1 grade, $\mathrm{TBx}+\mathrm{SBx}$ and $\mathrm{TBx}$ showed significantly higher concordance over SBx at RP (Figure 2 ). Concordance level was significantly higher for ISUP 1 in combined TBx + SBx method compared to conventional TRUS-Bx (61.3\% vs $37.9 \%, \mathrm{p}=0.014)$. In patients with biopsy ISUP 1 exclusively, significant upgrading was seen in TRUS-Bx (62.1\%) and SBx (55.5\%) when compared to TBx (41.4\%) and TBx+SBx (38.7\%). Upgrading rates for ISUP 2 were similar in SBx, TBx, TBx $+\mathrm{SBx}$. However, for ISUP 2 downgrading rates were significantly higher in $\mathrm{TBx}$ and $\mathrm{TBx}+\mathrm{SBx}$ when compared to $\mathrm{SBx}$ alone. For ISUP Grade 2, SBx and TRUS-Bx showed similar upgrading and downgrading rates. In ISUP 2, all biopsy methods showed similar upgrading but downgrading was higher in $\mathrm{TBx}$ and $\mathrm{TBx}+\mathrm{SBx}$ probably due to over sampling tersiyer 4 pattern in biopsy. Combined TBx $+\mathrm{SBx}$ showed significant downgrading in $47.8 \%$ and $54.6 \%$ for ISUP 3 and 4 respectively. For ISUP 3, 4, and 5 groups TBx, SBx, and TBx $+\mathrm{SBx}$ showed similar concordances. No upgrading was detected in SBx group for ISUP 3, 4 but downgrading was higher in SBx group when compared to $\mathrm{TBx}$ and $\mathrm{TBx}+\mathrm{SBx}$. For ISUP grade 2, TRUS-Bx showed the highest concordance among all biopsy methods (70\%) with less downgrading. For ISUP 2 and 3 TRUS-Bx showed superior but not 
significant concordance than combined $\mathrm{TBx}+\mathrm{SBx}$ method ( $70 \%$ vs $64.7 \% \mathrm{p}=0.509$ and $56.1 \%$ vs $43.5 \%$, $\mathrm{p}=0.332$ ). For ISUP 3 and 4, TRUS-Bx showed higher upgrading and less downgrading when compared to TBx + SBx. Conventional TRUS-Bx showed the worst concordance with $17.6 \%$ at ISUP Grade 4 with an upgrading rate of $47.1 \%$ which might be a reflection of inadequate sampling.

\section{Discussion}

The Gleason grading system has been shown to be the most important determinant of tumor aggressiveness, disease outcome, and mortality from prostate cancer (19). Gleason Score discordance can confound optimal treatment allocation of patients diagnosed with prostate cancer and place them at risk of worse oncological outcomes. MRI targeted biopsies, which utilise previously taken MRI images of suspected cancer and fuse them with real-time ultrasound images, are reported to be able to detect high grade cancers better than systematic biopsies and TRUS (6-9, 20,21). However, several recent studies have reported a mismatch in the biopsy and radical prostatectomy Gleason Score $(10,11,22)$. Despite the improved detection of clinically significant cancers with MRI targeted biopsies, considerable disparity exists in the literature regarding MRI targeted biopsy's ability to better detect RP pathology in biopsy-naive men over conventional TRUS biopsies $(10,11,22)$. We performed this study in order to investigate if targeted prostate biopsy has a performance superior to untargeted biopsy in determining the optimal agreement between biopsy and surgical Gleason Score. Our findings suggest that in the overall patient cohort, combined $\mathrm{TBx}+\mathrm{SBx}$ provides improved detection rates particularly for higher-grade disease over either systematic or MRI-targeted biopsy or TRUS$\mathrm{Bx}$ alone. However, superiority in significant cancer detection did not reflect accurate Gleason Grade Group in the whole prostate gland at final surgical pathology due to selective sampling of higher grade areas within an otherwise low grade cancer. MRI targeted biopsy was better in the prediction of the result of final histopathological analysis for ISUP 1 and ISUP 4 than systematic biopsies or TRUS-Bx. Our analysis of biopsy techniques according to ISUP Grade groups showed increased downgrading in MRI fusion biopsy and increased upgrading in TRUS-Bx.

In this cohort of patients, combined TBx $+\mathrm{SBx}$ biopsy provided more accurate diagnosis than MRI targeted or systematic biopsy alone. Consistent with earlier studies, we found higher cancer detection rates on TBx when compared to $\operatorname{SBx}(7,9,23)$. However, 13 out of 127 prostate cancers $(10.2 \%)$ were missed by TBx alone. Missed cancers by TBx may reflect the underlying limitation of mpMRI which might result from factors such as PCa not visible on mpMRI, varying inter-reader agreement on the mpMRI results and/or missing the target lesion in biopsy.

Our results have shown that MRI targeted biopsies are significantly better for the detection of clinically significant PCa (ISUP 2 or higher) than TRUS-Bx. Almost half of the cancers detected by TRUS-Bx were in ISUP grade 1 category. When TBx alone is compared to SBx alone for the detection rate of significant prostate cancer diagnosis, overall targeted biopsy alone tends to detect more significant PCa but this superiority does not reach statistical difference. In the present study, SBx had superior performance to TRUS-Bx in determining high grade cancers. This could be a result of the cognitive fusion bias within the $\mathrm{SBx}$ that the urologist performing the $\mathrm{SBx}$ was aware of the localization of the suspicious lesion on mpMRI. In our study, if a pure TBx strategy omitting SBx is applied, this will lead to missing significant prostate cancer in 11 patients. Our results support the notion that in order to obtain the most accurate assessment of the entire prostate gland especially for patients at risk of significant disease, SBx remains necessary, in addition to the TBx due to limitations of mpMRI performance/reading and of precision during lesion targeting.

In this study, combined $\mathrm{TBx}+\mathrm{SBx}$ showed significantly better concordance than any biopsy method alone in ISUP Grade 1. Also, combined TBX $+\mathrm{SBx}$ prostate biopsy resulted in significantly less pathologic upgrading in ISUP 1 grade group as compared with TRUS-Bx at prostatectomy ( $38.7 \%$ vs $62.1 \%$ ). Men under active surveillance in ISUP Grade 1 group diagnosed by TRUS-Bx are at significantly greater risk of subsequent reclassification by confirmatory biopsies. It has been reported that up to $40 \%$ of men potentially suitable for AS had unfavorable disease at RP and these high rates of adverse pathologic findings which can underestimate aggressiveness of the disease (24-26). Upgrading is particularly a great concern in the 
context of active surveillance. Pathologic upgrading has been shown to be associated with adverse outcomes, including higher rates of biochemical recurrence $(27,28)$. A recent study by our group has shown that men suitable for AS, but elected immediate RP, proved to have a GS upgrade rate of $30.6 \%$ and a pathological upstaging rate of $13.2 \%$ (29). In the present study, our results clearly suggest that TRUS-Bx based active surveillance decision should be questioned unless confirmatory mpMRI $\mathrm{TBx}+\mathrm{SBx}$ is done. In the active surveillance scenario, it is imperative to decrease the risk of missing a high-grade disease and delaying a radical treatment, providing more confidence to the urologist and the patient with conservative management of PCa. Based on our data, multiparametric MRI TBx $+\mathrm{SBx}$ is the best available strategy to stratify men to AS with reliable eligibility and should be included in the AS protocols for a more accurate grading of PCa.

Among all biopsy methods, the lowest concordance was achieved in ISUP Grade 4. Combined TBx $+\mathrm{SBx}$ showed significantly better concordance than TRUS-Bx in ISUP Grade 4. At this group, TRUS-Bx showed increased upgrading $(47.1 \%)$ but in contrast TBx + SBx showed increased downgrading (54.6\%). Downgrading may be due to oversampling that small foci of Gleason 5 detected at biopsy but not documented at radical prostatectomy (4). In ISUP Grade 4, SBx was better than TRUS-Bx. This, again, shows that inadequate diagnostic biopsy sampling is high in TRUS-Bx and most of the SBx are not blind and affected by MRI cognition.

Our study has demonstrated that TRUS-Bx concordance was superior for ISUP 2,3 and 5 Grade Groups than TBx + SBx. In all ISUP groups except 1 and 4, TRUS-Bx showed better performance in predicting final pathology with higher concordance. MRI Targeted biopsies are more prone to downgrading and TRUS biopsies are more upgraded. In the present study, downgrading was higher in $\mathrm{TBx}+\mathrm{SBx}$ biopsy group compared to conventional TRUS-Bx. Downgrading may occur for several reasons, including over-sampling of the very small (less than 5\%) foci of Gleason pattern 4 or 5 cancer $(4,30)$. There is a risk of oversampling with increased number of targets registered by radiologist and increased number of biopsy cores taken at discretion of the biopsy performer. A very small foci of Gleason pattern 4 or 5 tumor might be missed by the routine radical prostatectomy pathologic examination or might be noted as the presence of a tertiary Gleason pattern if these small tumors are detected. It is reported that the issue of accounting for tertiary grade patterns is significant as can be seen in almost $20 \%$ of RP specimens (4). An increased number of target lesions on mpMRI and thus,an increased number of core biopsy per lesion may cause needle biopsy to sample a tertiary higher grade pattern in the RP, which is not recorded in the standard GS reporting, resulting in apparent overgrading on the needle biopsy. Our results suggest that if the physician is assigning the patient as high risk group solely based on biopsy pathology, there would be a risk of overtreating quite a few patients. Having increased the percentage of surgical pathology downgrading, MRI fusion biopsy implies a high rate of overdiagnosis of Gleason sum 8 scores on biopsy, potentially leading to suboptimal treatment strategies and patient distress.

Although conventional TRUS-Bx is a blind, nontargeted and not always anatomically systematic biopsy, our findings portray that TRUS-Bx is superior but not yet significant over SBx in accurate prediction of final pathology. However, there is a difference of $7.6 \%$ and $22.4 \%$ for ISUP 1 and 4 grades between the schemes respectively favoring SBx. This may be a result of cognitive impact of MRI findings that the unblind nature of systematic biopsies after mpMRI were probably contaminated by MRI findings and not even described to be so, in practice it influences and orients biopsy.

The major limitations of our study are its retrospective nature and analysis. Another limitation is that TBx data was obtained from 5 different centers performed by 7 different urologists using different MRI and MRIUS Fusion devices. Failure of mpMRI fusion biopsy due to mpMRI incorrect image registration or mismatch of image planes, inaccurate sampling and intralesional Gleason Score heterogeneity may have impacted our results. Another important limitation is that there was no centralized pathological examination, multicentric pathological examinations by uropathologists at respective centers. However, our data reflects the real-life nationwide picture.

In conclusion, our results have shown that MRI-targeted biopsies are significantly better for the detection of clinically significant PCa (ISUP 2 or higher) than TRUS-Bx. However, superiority in significant cancer 
detection appears at least in part as a result of oversampling of higher grade areas within an otherwise low grade cancer and may not reflect accurate Gleason Grade Group in the whole prostate gland at final surgical pathology due to increased downgrading. There is a risk of overtreatment if biopsy Gleason Group was taken as a unique parameter for treatment decision. TRUS-Bx has superior concordance with radical prostatectomy for ISUP 2,3,5 grade groups, but increased upgrading should also be taken into account leading to undertreatment. MRI Targeted biopsies combined with systematic biopsies has the greatest concordance in ISUP Grade 1 which is of utmost importance for active surveillance indicating that active surveillance decision should be based on MRI targeted + systematic biopsies either at initial diagnosis or in confirmatory biopsy.

\section{References}

1. De Rooij M, Hamon EH, Futterer JJ, Barentsz JO and Rovers MM: Accuracy of multiparametric MRI for prostate cancer detection: a meta analysis. AJR Am J Roentgenol 202: 343-351, 2014.

2. Siddiqui MM, Rais-Bahrami S, Turkbey B, et al. Comparison of MR/ultrasound fusion-guided biopsy with ultrasound-guided biopsy for the diagnosis of prostate cancer. JAMA 313: 390-397, 2015

3. Cohen MS, Hanley RS, Kurteva T, et al. Comparing the Gleason prostate biopsy and Gleason prostatectomy grading system: the Lahey Clinic Medical Center experience and an international meta-analysis. Eur Urol. 2008;54:371-81.

4. Epstein JI, Feng Z, Trock BJ, Pierorazio PM. Upgrading and downgrading of prostate cancer from biopsy to radical prostatectomy: incidence and predictive factors using the modified Gleason grading system and factoring in tertiary grades. Eur Urol 2012; 61: 1019-24.

5. Alchin DR, Murphy D, Lawrentschuk N. Risk factors for Gleason score upgrading following radical prostatectomy. Minerva Urol Nefrol 2017 ;69: 459-465.

6. Diamand R, Oderda M, Al Hajj Obeid W, et al. A multicentric study on accurate grading of prostate cancer with systematic and MRI/US fusion targeted biopsies: comparison with final histopathology after radical prostatectomy. World J Urol. 2019 Oct;37(10):2109-2117. doi: 10.1007/s00345-019-026349. Epub 2019 Jan 16.

7. Ahdoot M, Wilbur AR, Reese SE, et al. MRI-Targeted, Systematic, and Combined Biopsy for Prostate Cancer Diagnosis. N Engl J Med. 2020 Mar 5;382(10):917-928.

8. Ahmed HU, El-Shater Bosaily A, Brown LC, et al. Diagnostic accuracy of multi-parametric MRI and TRUS biopsy in prostate cancer (PROMIS): a paired validating confirmatory study. Lancet. (2017) 389:815-22. doi: 10.1016/S0140-6736(16)32401-1

9. Kasivisvanathan V, Rannikko AS, Borghi M, et al. MRI-targeted or standard biopsy for prostatecancer diagnosis. N Engl J Med. 2018; 378:1767-77.

10. Ruhrup J, Preisser F, Theissen L, et al. MRI-Fusion Targeted vs. Systematic Prostate Biopsy-How Does the Biopsy Technique Affect Gleason Grade Concordance and Upgrading After Radical Prostatectomy? Front Surg. 2019 Sep 18;6:55. doi: 10.3389/fsurg.2019.00055. eCollection 2019.

11. Goel S, Shoag JE, Gross MD, et al. Concordance Between Biopsy and Radical Prostatectomy Pathology in the Era of Targeted Biopsy: A Systematic Review and Meta-analysis. Eur Urol Oncol. 2020 Feb;3(1):10-20. doi: 10.1016/j.euo.2019.08.001. Epub 2019 Sep 4.

12. Porpiglia F, DE Luca S, Passera R, et al. Multiparametric Magnetic Resonance/Ultrasound Fusion Targeted Biopsy Improves Agreement Between Biopsy and Radical Prostatectomy Gleason Score. Anticancer Res. 2016; 36: 4883-9.

13. Borkowetz A, Platzek I, Toma M, et al. Direct comparison of multiparametric magnetic resonance imaging (MRI) results with final histopathology in patients with proven prostate cancer in MRI/ultrasonography-fusion biopsy. BJU Int. 2016 Aug;118(2):213-20.

14. D. Lea J, Stephensond S, Bruggerd M, et al. MRI-ultrasound fusion biopsy for prediction of final prostate pathology. J Urol. 2014 November ; 192(5): 1367-1373.

15. Weinreb JC, Barentsz JO, Choyke PL, et al. PI-RADS Prostate Imaging - Reporting and Data System: 2015, version 2. Eur Urol 2016; 69: 16-40.

16. Epstein JI, Egevad L, Amin MB, et al.The 2014 International Society of Urological Pathology (ISUP) 
Consensus Conference on Gleason Grading of Prostatic Carcinoma: definition of grading patterns and proposal for a new grading system. Am J Surg Pathol. 2016; 40(2):244-52.

17. Harris PA, Taylor R,Thielke R, Payne J, Gonzalez N, Conde JG. Research electronic data capture (REDCap) - A metadata-driven methodology and workflow process for providing translational research informatics support. J Biomed Inform. 2009;42(2):377-81.

18. Harris PA,Taylor R, Minor BL, et al. REDCap Consortium, The REDCap consortium: Building an international community of software partners. J Biomed Inform. 2019 May 9 [doi: 10.1016/j.jbi.2019.103208]

19. Egevad L, Granfors T, Karlberg L, Bergh A, Stattin P. Prognostic value of the Gleason score in prostate cancer. BJU Int 2002; 89: 538-542.

20. van Luijtelaar A, Bomers J, Futterer J. A comparison of magnetic resonance imaging techniques used to secure biopsies in prostate cancer patients. Expert Rev Anticancer Ther. 2019 Aug;19(8):705-716.

21. Drost FH, Osses DF, Nieboer D, et al. Prostate MRI, with or without MRI-targeted biopsy, and systematic biopsy for detecting prostate cancer. Cochrane Database Syst Rev. 2019;4:CD012663.

22. Alshak MN, Patel N, Gross MD, Margolis D, Hu JC. Persistent Discordance in Grade, Stage and NCCN Risk Stratification in Men Undergoing targeted Biopsy and Radical Prostatectomy. Urology 2020; 135: 117-123.

23. Boesen L. Multiparametric MRI in detection and staging of prostate cancer. Dan Med J. 2017 Feb;64(2). pii: B5327.

24. Drost FJH, Rannikko A, Valdagni R, et al. Can active surveillance really reduce the harms of overdiagnosing prostate cancer? A reflection of real life clinical practice in the PRIAS study. Transl Androl Urol 2018; $7: 98-105$.

25. Radtke JP, Kuru TH, Bonekamp D, et al. Further reduction of disqualification rates by additional MRI-targeted biopsy with transperineal saturation biopsy compared with standard 12-core systematic biopsies for the selection of prostate cancer patients for active surveillance. Prostate Cancer Prostatic Dis, 2016; 19: 283-291.

26. Berglund RK, Masterson TA, Vora KC, Eggener SE, Eastham JA, Guillonneau BD. Pathological upgrading and up staging with immediate repeat biopsy in patients eligible for active surveillance. J Urol. 2008;180:1964-1967.

27. Musunuru HB, Yamamoto T, Klotz L, et al. Active surveillance for intermediate risk prostate cancer: survival outcomes in the sunnybrook experience. J Urol. 2016;196:1651-1658.

28. Patel HD, Tosoian JJ, Carter HB, Epstein JI. Adverse pathologic findings for men electing immediate radical prostatectomy: defining a favorable intermediate-risk group. JAMA Oncol. 2018;4:89-92.

29. Tinay I, Aslan G, Kural AR, et al. Pathologic Outcomes of Candidates for Active Surveillance Undergoing Radical Prostatectomy: Results from a Contemporary Turkish Patient Cohort. Urol Int. 2018;100:43-49.

30. Gondo T, Poonc BY, Matsumotoa K, Bernsteina M, Sjobergc DD, Easthama JA. Clinical role of pathological downgrading after radical prostatectomy in patients with biopsy-proven Gleason score $3+4$ prostate cancer. BJU Int. 2015 January ; 115: 81-86.

\section{Legend to Figures}

Figure 1. Concordance, upgrade and downgrade ratios between Combined TBx $+\mathrm{SBx}$ and Conventional TRUS-Bx method.

Figure 2. Concordance, upgrading and downgrading of biopsy compared to RP ISUP grades stratified by biopsy approach.

\section{Hosted file}

Table 1.pdf available at https://authorea.com/users/363643/articles/484347-comparison-oftrus-and-combined-mri-targeted-plus-systematic-prostate-biopsy-for-the-concordancebetween-biopsy-and-radical-prostatectomy-pathology 


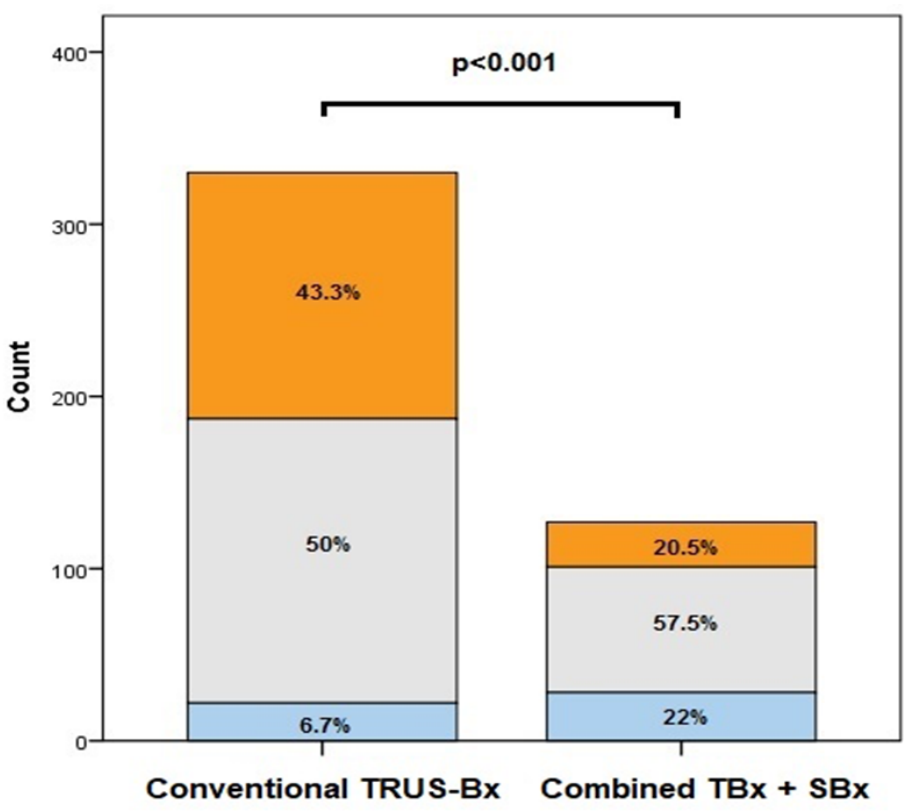

$\square$ Upgrade
$\square$ Concordance
$\square$ Downgrade

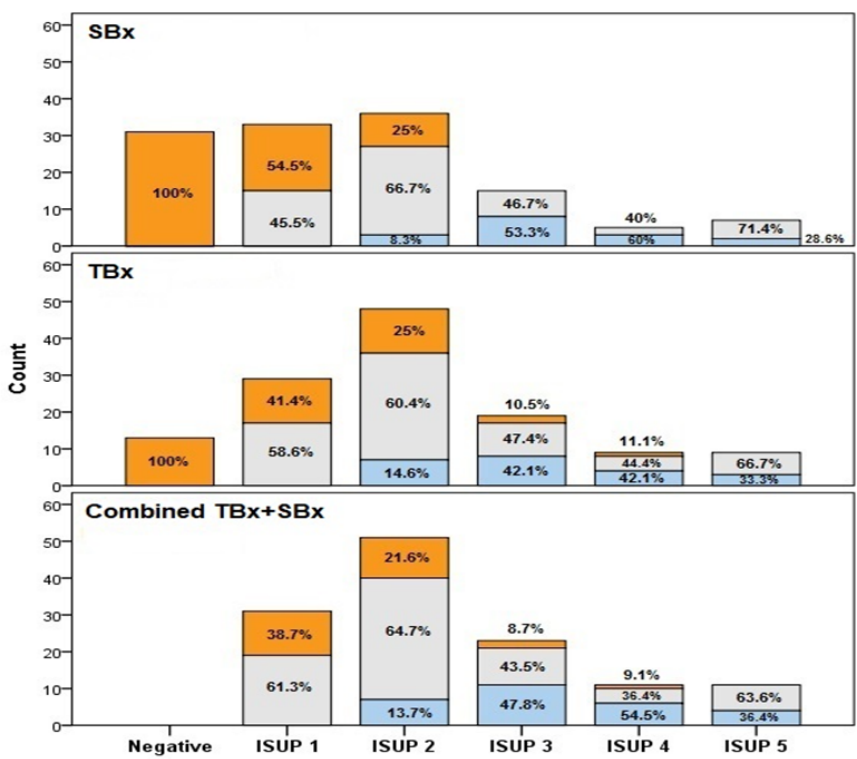

Upgrade

Concordan

Downgrade 